\title{
The Effect of Cold Ischemic Time on Immunohistochemical Evaluation of Invasive Ductal Carcinoma
}

\author{
Hye Jin Kim ${ }^{1}$, Han-Byoel Lee ${ }^{1}$, Tae-Kyung Yoo ${ }^{1}$, Jongjin Kim², Young-Joon Kang ${ }^{1}$, Jaihong Han ${ }^{1}$, Yumi Kim ${ }^{1}$, \\ Hyeong-Gon Moon ${ }^{1,3}$, Wonshik Han ${ }^{1,3}$, Dong-Young Noh ${ }^{1,3}$ \\ ${ }^{1}$ Department of Surgery, Seoul National University College of Medicine, Seoul; ${ }^{2}$ Department of Surgery, Boramae Medical Center, Seoul; ${ }^{3}$ Cancer \\ Research Institute, Seoul National University College of Medicine, Seoul, Korea
}

Purpose: Immunohistochemical $(\mathrm{IHC})$ evaluation is important for the management of breast cancer. Cold ischemic time (CIT) may result in inaccurate IHC results. The aim of this study was to investigate the effect of CIT on IHC results of invasive ductal carcinoma (IDC). Methods: We retrospectively reviewed the records of 316 patients diagnosed with IDC from February 2013 to June 2013 at a single hospital in Korea. The clinicopathological characteristics and IHC positivity for the estrogen receptor (ER), progesterone receptor (PR), Ki-67, and human epidermal growth factor receptor 2 (HER2) were compared between specimens with a CIT of $\leq 3$ hours (short CIT) and those with a CIT of $>12$ hours (long CIT). Results: Among the 316 patients, 227 had a CIT of $\leq 3$ hours and 89 had a CIT of $>12$ hours. No difference in positivity for ER $(p=0.734), \operatorname{PR}(p=0.870)$, and HER2 $(p=0.830)$ was observed between the two groups. In ER-positive specimens, the mean percentage value was higher in the long CIT group than in the short CIT group $(81.3 \pm 20.8 \%$ vs. $76.0 \pm 26.3 \%$, respectively; $p=0.021)$. The mean $\mathrm{Ki}-67$ value was higher in the long CIT group compared to the short CIT group ( $9.7 \pm 14.6 \%$ vs. $7.0 \pm 10.8 \%$, respectively; $p=0.047)$. Conclusion: $\mathrm{CIT}$ did not affect the positivity of ER, PR, HER2, and Ki-67 as determined by IHC when stored at $4^{\circ} \mathrm{C}$ overnight. However, specimens with a long $\mathrm{CIT}$ had a higher ER percentage value in ER-positive tumors and a higher Ki-67 value in all specimens compared to those with a short $\mathrm{CIT}$. These differences should be considered in the interpretation of IHC results in specimens with a long CIT.

Key Words: Breast neoplasms, Cold ischemia, Immunohistochemistry

\section{INTRODUCTION}

Breast cancer is known for its well-established prognostic clinicopathological features such as age, tumor size, nodal status, grade, hormone receptor status, and human epidermal growth factor receptor 2 (HER2) status. Since the monumental meta-analysis of tamoxifen by the Early Breast Cancer Trialists' Collaborative Group in 1998 [1], the introduction of antihormonal therapy has revolutionized breast cancer treatment, thereby resulting in better survival outcomes. In addition, after the approval of trastuzumab by the Food and Drug Admin-

\section{Correspondence: Dong-Young Noh}

Department of Surgery and Cancer Research Institute, Seoul National University College of Medicine, 101 Daehak-ro, Jongno-gu, Seoul 03080, Korea

Tel: +82-2-2072-2921, Fax: +82-2766-3975, E-mail: dynoh@snu.ac.kr

This work was supported by grant from the Korean Healthcare Technology R\&D project through the Korean Health Industry Development Institute (KHIDI), funded by the Ministry of Health \& Welfare, Republic of Korea (grant number: HI13C2148 and HI14C1277).

Received: October 6, 2015 Revised: October 23, 2015

Accepted: November 21, 2015 istration in 2006 as part of an adjuvant treatment regimen for women with node-positive, HER2-overexpressing breast cancer, the target therapy agent has had a major impact in the treatment of HER2-positive breast cancer [2]. Immunohistochemical (IHC) evaluation of tumors is essential to guide treatment decisions using these therapeutic agents. Hormonal markers such as estrogen receptor (ER), progesterone receptor (PR), and markers of apoptosis and cell proliferation including Ki-67, bcl-2, and p53, as well as HER2, are common biomarkers evaluated by IHC $[3,4]$.

The recent development of molecular diagnostic tools such as Oncotype DX ${ }^{\circledR}$ (Genomic Health, Redwood City, USA) has provided additional prognostic information for systemic therapy decisions. However, a prognostic model developed using the four IHC measurements of ER, PR, HER2, and Ki-67 has been shown to provide enough prognostic information, similar to that provided by Oncotype DX ${ }^{\circledR}$ for endocrine-treated ER-positive breast cancer patients [5]. Considering the cost and delay in treatment resulting from molecular testing, classical IHC evaluation of formalin-fixed paraffin-embedded tissues 
may provide a more cost-effective way of making systemic therapy decisions, especially in Korea.

The accuracy of IHC staining is important for accurate evaluation of the biomarkers. Improvement guidelines for the standardization of assays have been provided by The American Society of Clinical Oncology and College of American Pathologists (ASCO/CAP) for HER2, ER, and PR [6,7]. According to the guidelines, the cold ischemic time (CIT) should be limited to less than 1 hour, and the samples should be fixed in $10 \%$ neutral buffered formalin for not less than 6 hours and not more than 72 hours for ER, PR, and HER2 testing.

In large-volume hospitals in Korea in which more than 1,500 breast cancer operations are performed every year, there is concern for timely fixation of tissues according to the ASCO/CAP guidelines. The delay in time from resection of breast tissue to fixation, or prolonged CIT, may affect the outcome of IHC biomarkers, and in turn, the therapeutic decisions made by physicians. The aim of this study was to investigate the effect of CIT on the IHC results of invasive ductal carcinoma (IDC).

\section{METHODS}

This retrospective study was approved by the Institutional Review Board of Seoul National University Hospital (IRB number: 1306-006493) and conducted in accordance with the Declaration of Helsinki. The requirement for informed consent was waived.

\section{Patients and variables}

We retrospectively reviewed the records of 316 consecutive patients who underwent breast surgery for IDC from February 2013 to June 2013 at Department of Surgery of Seoul National University Hospital. Patients who had received neoadjuvant chemotherapy were excluded. Data about clinicopathological characteristics including age, type of surgery, tumor size, nodal status, nuclear grade, histologic grade, and pathologic stage were collected and analyzed. IHC staining was performed using the avidin-biotin complex technique for staining 4- $\mu \mathrm{m}$ thick tissue sections from paraffin-embedded tissue blocks. The evaluation of ER, PR, HER2, and Ki-67 expression was performed by the same pathologist. The ER, PR, and HER2 status was determined according to the ASCO/CAP guidelines [8,9]. A high expression of Ki67 was defined as expression in $\geq 10 \%$ of tumor tissues, as the $10 \%$ cutoff provided the best predictive value for prognosis in our institution [10]. The methods used in this study are summarized in Figure 1.

\section{Definition of CIT}

The surgically resected specimens were divided into two groups according to the CIT as determined by the length of time from the end of surgery to tissue fixation. The short CIT and long CIT groups were defined as CIT of $\leq 3$ hours and CIT of $>12$ hours, respectively. The difference in CIT was mainly determined by whether the surgical specimen was formalin-fixed on the same day as the operation (CIT $\leq 3$ hours) or on the following day (CIT $>12$ hours) after being stored overnight in a refrigerator at $4^{\circ} \mathrm{C}$.

\section{Statistical analysis}

The chi-square test was used to observe the associations between the length of CIT and other variables. A Student t-test was performed to assess whether age, ER, PR, and Ki-67 values were associated with the length of CIT. $p$-values $<0.05$ were considered statistically significant. Statistical analyses were performed with SPSS statistical software, version 22 (SPSS Inc., Armonk, USA).

\section{Breast surgery for invasive ductal carcinoma}

Seoul National University Hospital Breast Care Center February 2013-June 2013

Neoadjuvant chemotherapy patients excluded

\section{Retrospective chart review of 316 patients}

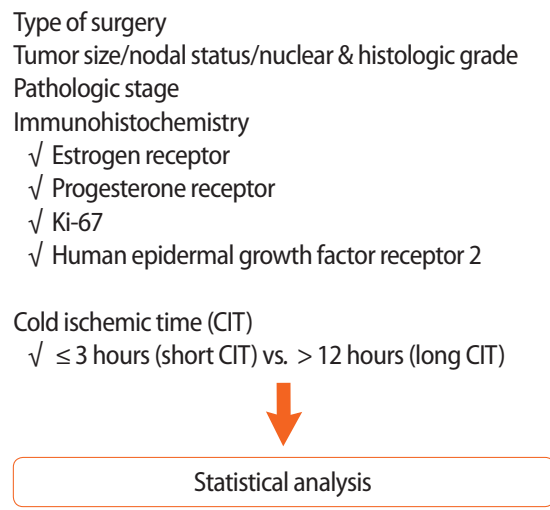

Student t-test/chi-square test

Figure 1. Summary of methods used in this study. The clinicopathological variables of patients who underwent breast surgery for invasive ductal carcinoma were analyzed according to the length of cold ischemic time. 
Table 1. Clinicopathological characteristics according to length of cold ischemic time

\begin{tabular}{|c|c|c|c|}
\hline Characteristic & $\begin{array}{c}\text { Short CIT }(n=227) \\
\text { No. }(\%)\end{array}$ & $\begin{array}{c}\text { Long CIT }(n=89) \\
\text { No. }(\%)\end{array}$ & $p$-value \\
\hline Age $(y r)^{*}$ & $51.7 \pm 9.6$ & $49.7 \pm 11.0$ & 0.043 \\
\hline Tumor size $(\mathrm{cm})$ & & & 0.389 \\
\hline$\leq 2$ & $132(58.1)$ & $47(52.8)$ & \\
\hline$>2$ & 95 (41.9) & $42(47.2)$ & \\
\hline Nodal status & & & 0.623 \\
\hline Negative & 149 (65.6) & $61(68.5)$ & \\
\hline Positive & $78(34.4)$ & $28(31.5)$ & \\
\hline Stage & & & 0.754 \\
\hline 0 & $2(0.9)$ & 0 & \\
\hline 1 & $108(47.6)$ & $45(50.6)$ & \\
\hline$\|$ & $92(40.5)$ & $33(37.1)$ & \\
\hline III & $25(11.0)$ & $11(12.3)$ & \\
\hline Nuclear grade & & & 0.990 \\
\hline 1,2 & $92(40.5)$ & $36(40.4)$ & \\
\hline 3 & $135(59.5)$ & $53(59.6)$ & \\
\hline Histologic grade & & & 0.766 \\
\hline 1,2 & $119(52.4)$ & 45 (50.6) & \\
\hline 3 & $108(47.6)$ & 44 (49.4) & \\
\hline \multicolumn{4}{|l|}{$\mathrm{IHC}$} \\
\hline ER & & & 0.734 \\
\hline Negative & $57(25.1)$ & $24(27.0)$ & \\
\hline Positive & $170(74.9)$ & $65(73.0)$ & \\
\hline PR & & & 0.870 \\
\hline Negative & $87(38.3)$ & $35(39.3)$ & \\
\hline Positive & $140(61.7)$ & $54(60.7)$ & \\
\hline HER2 & & & 0.830 \\
\hline Negative & $186(81.9)$ & $72(80.9)$ & \\
\hline Positive & 41 (18.1) & $17(19.1)$ & \\
\hline Ki-67 (\%) & & & 0.247 \\
\hline$<10$ & $191(84.1)$ & 70 (78.7) & \\
\hline$\geq 10$ & $36(15.9)$ & $19(21.3)$ & \\
\hline Type of surgery & & & 0.011 \\
\hline Mastectomy & $61(26.9)$ & 37 (41.6) & \\
\hline $\mathrm{BCS}$ & $166(73.1)$ & $52(58.4)$ & \\
\hline
\end{tabular}

$\mathrm{CIT}=$ cold ischemic time; $\mathrm{IHC}=$ immunohistochemistry; $\mathrm{ER}=$ estrogen receptor; $\mathrm{PR}=$ progesterone receptor; $\mathrm{HER2}=$ human epidermal growth factor receptor 2; BCS = breast-conserving surgery.

*Mean \pm SD.

\section{RESULTS}

Among the 316 patients, 227 patients $(71.8 \%)$ had a CIT of $\leq 3$ hours (short CIT) and 89 patients (28.2\%) had a CIT of $>12$ hours (long CIT). The clinicopathological characteristics according to the length of CIT are summarized in Table 1. The mean age was slightly higher in the short CIT group ( $51.7 \pm 9.6$ years vs. $49.7 \pm 11.0$ years in the long CIT group, $p=0.043$ ). There was no statistical difference in tumor size between the groups when stratified into sizes of $\leq 2 \mathrm{~cm}$ and $>2 \mathrm{~cm}$.
Table 2. Estrogen receptor, progesterone receptor, and Ki-67 values according to length of cold ischemic time

\begin{tabular}{lccc}
\hline & $\begin{array}{c}\text { Short CIT }(n=227) \\
\text { Mean } \%\end{array}$ & $\begin{array}{c}\text { Long CIT }(n=89) \\
\text { Mean } \%\end{array}$ & $p$-value \\
\hline ER & & & \\
All & $57.0 \pm 40.1$ & $59.4 \pm 40.4$ & 0.905 \\
Positive & $76.0 \pm 26.3$ & $81.3 \pm 20.8$ & 0.021 \\
PR & & \\
All & $26.4 \pm 38.9$ & $31.6 \pm 37.7$ & 0.056 \\
Positive & $42.9 \pm 35.6$ & $52.2 \pm 35.7$ & 0.895 \\
Ki-67 (\%) & & & \\
All & $7.0 \pm 10.8$ & $9.7 \pm 14.6$ & 0.047 \\
$<10$ & $3.5 \pm 2.9$ & $4.2 \pm 3.1$ & 0.279 \\
$\geq 10$ & $25.2 \pm 17.3$ & $29.8 \pm 21.4$ & 0.600 \\
\hline CIT = cold ischemic time; ER = estrogen receptor; $P R=$ progesterone receptor.
\end{tabular}

The nodal status, pathologic stage, and nuclear/histologic grade were also statistically similar between the groups. Mastectomy was performed at a lower rate in the short CIT group (26.9\% vs. $41.6 \%$ in the long CIT group, $p=0.011$ ). There was no difference in ER and PR positivity between the groups ( $E R, p=0.734 ; \mathrm{PR}, p=0.870$ ). No difference was observed in the HER2 status according to ASCO/CAP guidelines and Ki-67 using the $10 \%$ cutoff value (HER2, $p=0.830$; Ki- 67 , $p=0.247)$.

On analysis of the ER, PR, and Ki-67 percentage values according to their positivity and length of CIT, differences were observed in specific subgroups (Table 2). The mean ER percentage values in all the patients showed no difference between the short and long CIT groups $(57.0 \pm 40.1 \%$ vs. $59.4 \pm 40.4 \%$, respectively; $p=0.905)$. However, in ERpositive patients, the mean value was significantly higher in the long CIT group $(76.0 \pm 26.3 \%$ vs. $81.3 \pm 20.8 \%$ in the short CIT group, $p=0.021$ ). Ki-67 values were observed to be higher in the long CIT group $(9.7 \pm 14.6 \%$ vs. $7.0 \pm 10.8 \%$ in the short CIT group, $p=0.047)$, whereas no differences were observed in the subgroups for Ki-67 $<10 \%(4.2 \pm 3.1 \%$ vs. $3.5 \pm 2.9 \%, p=0.279)$ and Ki- $67 \geq 10 \%(29.8 \pm$ $21.4 \%$ vs. $25.2 \pm 17.3 \%, p=0.600$ ). There was no significant difference in the PR percentage values between the two groups, although a comparison between all patients tended to have a higher PR value in the long CIT group ( $31.6 \pm 37.7 \%$ vs. $26.4 \pm 38.9 \%$ in the short CIT group, $p=0.056$ ).

\section{DISCUSSION}

In this study, we reviewed the records of 316 consecutive patients 
with IDC and evaluated whether the length of CIT influences IHC findings. It was observed that CIT, when divided into groups of $\leq 3$ hours and $>12$ hours, does not affect the positivity of the IHC biomarkers ER, PR, HER2, and Ki-67. However, the percentage values of ER in ER-positive tumors and Ki-67 values in all tumors were greater in specimens with a long CIT.

The results of many previous studies [11-14] are in agreement with those of our study, where IHC profile from specimens stored overnight in a refrigerator at $4^{\circ} \mathrm{C}$ and formalin-fixed on the day after surgery did not alter treatment decisions. Pinhel et al. [11] compared corecut specimens that were formalin-fixed immediately and resected specimens that had a delay in time to fixation, and observed no significant difference in the positivity for ER, PR, and HER2. In another study [12], the authors stored an invasive lobular carcinoma specimen in a fresh state without any fixative in the refrigerator at $4^{\circ} \mathrm{C}$ for 4 days to assess the effect of prolonged CIT. The tumor showed no degradation and no differences in the quality of tissue for ER and PR IHC staining. The accuracy of HER2 was investigated by analyzing the HER2 IHC and fluorescence in situ hybridization results and was shown to remain accurate considering the ASCO/CAP guidelines $[13,14]$.

However, the results of other studies $[15,16]$ have supported standardization of CIT and strict compliance to the ASCO/CAP guidelines. Prolonged tumor ischemia that is induced by delays in formalin fixation is known to cause decreased IHC accuracy by destroying tissue protein expression, especially ER and PR. In a study by Khoury et al. [15], the effects of delayed formalin fixation on 10 specimens that were kept at room temperature for $0,10,30$ minutes, and 1, 2, 4, and 8 hours before fixation were explored. The authors concluded that ER expression began to decline after 2 hours and PR expression began to decline after 1 hour of CIT. After 8 hours of CIT, ER expression was completely negative, and they recommended that specimens be fixed ideally within 1 hour of receipt and not be stored overnight at $4^{\circ} \mathrm{C}$. In another study by Qiu et al. [16], delayed formalin fixation and associated long CIT were negatively correlated with the hormone receptor expression in diagnostic specimens, such as surgical excision specimens. A decreased protein level by delayed fixation was initially shown on ligand binding assays and a progressive delay of fixation was correlated with a progressive decrease of both the percentage and intensity of ER, PR, and HER2 IHC staining of breast tumor cells.
Our results showed an increase in percentage values of ER in ERpositive tumors and $\mathrm{Ki}-67$ in all tumors with a long CIT. These findings have not been observed in previous studies [15,16], where most showed a decrease in values. This may be explained by the fact that specimens with a long CIT were put in the refrigerator at $4^{\circ} \mathrm{C}$ with short exposure to room temperature, whereas some specimens with a short CIT were stored at room temperature for as long as 3 hours before being fixed. This may support the findings of previous studies where the IHC profile of refrigerated specimens was not affected by a long CIT [11-14]. Furthermore, a wide range of CIT in the short CIT group may have prevented specific evaluation of specimens with CIT of $\leq 1$ hour.

Some limitations of this study are the retrospective nature of data collection and the small number of specimens evaluated. In addition, the definition of CIT is arbitrary, being judged by whether the specimen was fixed on the operation day or the following day. Further prospective, large-scale studies with detailed grouping of CIT are needed for a more accurate evaluation of the effect of CIT on IHC findings.

In conclusion, CIT did not affect the positivity of ER, PR, HER2, and $\mathrm{Ki}-67$ as determined by IHC when stored at $4^{\circ} \mathrm{C}$ overnight. However, specimens with a long CIT had a higher ER percentage value in ERpositive tumors and a higher Ki-67 value in all specimens compared to those with a short CIT. These differences should be considered in the interpretation of IHC results in specimens with a long CIT.

\section{CONFLICT OF INTEREST}

The authors declare that they have no competing interests.

\section{REFERENCES}

1. Early Breast Cancer Trialists' Collaborative Group. Tamoxifen for early breast cancer: an overview of the randomised trials. Lancet 1998;351:1451-67.

2. Tan AR, Swain SM. Ongoing adjuvant trials with trastuzumab in breast cancer. Semin Oncol 2003;30(5 Suppl 16):54-64.

3. Zaha DC. Significance of immunohistochemistry in breast cancer. World J Clin Oncol 2014;5:382-92.

4. Zhao L, Yang X, Khan A, Kandil D. Diagnostic role of immunohistochemistry in the evaluation of breast pathology specimens. 
Arch Pathol Lab Med 2014;138:16-24.

5. Cuzick J, Dowsett M, Pineda S, Wale C, Salter J, Quinn E, et al. Prognostic value of a combined estrogen receptor, progesterone receptor, Ki-67, and human epidermal growth factor receptor 2 immunohistochemical score and comparison with the Genomic Health recurrence score in early breast cancer. J Clin Oncol 2011;29:4273-8.

6. Hammond ME, Hayes DF, Dowsett M, Allred DC, Hagerty KL, Badve S, et al. American Society of Clinical Oncology/College of American Pathologists guideline recommendations for immunohistochemical testing of estrogen and progesterone receptors in breast cancer (unabridged version). Arch Pathol Lab Med 2010;134: e48-72.

7. Wolff AC, Hammond ME, Hicks DG, Dowsett M, McShane LM, Allison KH, et al. Recommendations for human epidermal growth factor receptor 2 testing in breast cancer: American Society of Clinical Oncology/College of American Pathologists clinical practice guideline update. J Clin Oncol 2013;31:3997-4013.

8. Hammond ME, Hayes DF, Dowsett M, Allred DC, Hagerty KL, Badve S, et al. American Society of Clinical Oncology/College Of American Pathologists guideline recommendations for immunohistochemical testing of estrogen and progesterone receptors in breast cancer. J Clin Oncol 2010;28:2784-95.

9. Wolff AC, Hammond ME, Schwartz JN, Hagerty KL, Allred DC, Cote RJ, et al. American Society of Clinical Oncology/College of American Pathologists guideline recommendations for human epidermal growth factor receptor 2 testing in breast cancer. J Clin Oncol 2007;25:118-45
10. Jung SY, Han W, Lee JW, Ko E, Kim E, Yu JH, et al. Ki-67 expression gives additional prognostic information on St. Gallen 2007 and Adjuvant! Online risk categories in early breast cancer. Ann Surg Oncol 2009;16:1112-21

11. Pinhel IF, Macneill FA, Hills MJ, Salter J, Detre S, A’hern R, et al. Extreme loss of immunoreactive $\mathrm{p}$-Akt and $\mathrm{p}$-Erk1/2 during routine fixation of primary breast cancer. Breast Cancer Res 2010;12:R76.

12. Apple S, Pucci R, Lowe AC, Shintaku I, Shapourifar-Tehrani S, Moatamed N. The effect of delay in fixation, different fixatives, and duration of fixation in estrogen and progesterone receptor results in breast carcinoma. Am J Clin Pathol 2011;135:592-8.

13. Moatamed NA, Nanjangud G, Pucci R, Lowe A, Shintaku IP, Shapourifar-Tehrani S, et al. Effect of ischemic time, fixation time, and fixative type on HER2/neu immunohistochemical and fluorescence in situ hybridization results in breast cancer. Am J Clin Pathol 2011;136:754-61.

14. Yildiz-Aktas IZ, Dabbs DJ, Bhargava R. The effect of cold ischemic time on the immunohistochemical evaluation of estrogen receptor, progesterone receptor, and HER2 expression in invasive breast carcinoma. Mod Pathol 2012;25:1098-105.

15. Khoury T, Sait S, Hwang H, Chandrasekhar R, Wilding G, Tan D, et al. Delay to formalin fixation effect on breast biomarkers. Mod Pathol 2009;22:1457-67.

16. Qiu J, Kulkarni S, Chandrasekhar R, Rees M, Hyde K, Wilding G, et al. Effect of delayed formalin fixation on estrogen and progesterone receptors in breast cancer: a study of three different clones. Am J Clin Pathol 2010;134:813-9. 